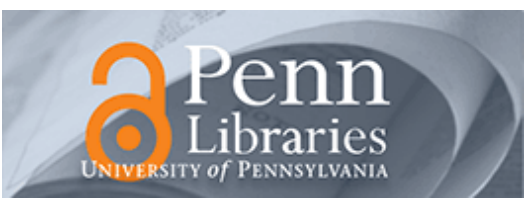

University of Pennsylvania

ScholarlyCommons

June 2005

\title{
Neuromorphic Implementation of Orientation Hypercolumns
}

\author{
Thomas Yu Wing Choi \\ Hong Kong University of Science and Technology \\ Paul A. Merolla \\ University of Pennsylvania \\ John V. Arthur \\ University of Pennsylvania \\ Kwabena A. Boahen \\ University of Pennsylvania, boahen@seas.upenn.edu \\ Bertram E. Shi \\ Hong Kong University of Science and Technology
}

Follow this and additional works at: https://repository.upenn.edu/be_papers

\section{Recommended Citation}

Choi, T. Y., Merolla, P. A., Arthur, J. V., Boahen, K. A., \& Shi, B. E. (2005). Neuromorphic Implementation of Orientation Hypercolumns. Retrieved from https://repository.upenn.edu/be_papers/62

Copyright 2005 IEEE. Reprinted from IEEE Transactions on Circuits and Systems--I: Regular Papers, Volume 52, Issue 6, June 2005, pages 1049-1060.

This material is posted here with permission of the IEEE. Such permission of the IEEE does not in any way imply IEEE endorsement of any of the University of Pennsylvania's products or services. Internal or personal use of this material is permitted. However, permission to reprint/republish this material for advertising or promotional purposes or for creating new collective works for resale or redistribution must be obtained from the IEEE by writing to pubs-permissions@ieee.org. By choosing to view this document, you agree to all provisions of the copyright laws protecting it.

This paper is posted at ScholarlyCommons. https://repository.upenn.edu/be_papers/62

For more information, please contact repository@pobox.upenn.edu. 


\title{
Neuromorphic Implementation of Orientation Hypercolumns
}

\begin{abstract}
Neurons in the mammalian primary visual cortex are selective along multiple stimulus dimensions, including retinal position, spatial frequency, and orientation. Neurons tuned to different stimulus features but the same retinal position are grouped into retinotopic arrays of hypercolumns. This paper describes a neuromorphic implementation of orientation hypercolumns, which consists of a single silicon retina feeding multiple chips, each of which contains an array of neurons tuned to the same orientation and spatial frequency, but different retinal locations. All chips operate in continuous time, and communicate with each other using spikes transmitted by the address-event representation protocol. This system is modular in the sense that orientation coverage can be increased simply by adding more chips, and expandable in the sense that its output can be used to construct neurons tuned to other stimulus dimensions. We present measured results from the system, demonstrating neuronal selectivity along position, spatial frequency and orientation. We also demonstrate that the system supports recurrent feedback between neurons within one hypercolumn, even though they reside on different chips. The measured results from the system are in excellent concordance with theoretical predictions.
\end{abstract}

\section{Keywords}

Address-event representation (AER), Gabor filter, image processing, mixed analog-digital integrated circuits, neural chips, neuromorphic engineering, visual cortex

\section{Comments}

Copyright 2005 IEEE. Reprinted from IEEE Transactions on Circuits and Systems--I: Regular Papers, Volume 52, Issue 6, June 2005, pages 1049-1060.

This material is posted here with permission of the IEEE. Such permission of the IEEE does not in any way imply IEEE endorsement of any of the University of Pennsylvania's products or services. Internal or personal use of this material is permitted. However, permission to reprint/republish this material for advertising or promotional purposes or for creating new collective works for resale or redistribution must be obtained from the IEEE by writing to pubs-permissions@ieee.org. By choosing to view this document, you agree to all provisions of the copyright laws protecting it. 


\title{
Neuromorphic Implementation of Orientation Hypercolumns
}

\author{
Thomas Yu Wing Choi, Paul A. Merolla, John V. Arthur, Kwabena A. Boahen, and Bertram E. Shi
}

\begin{abstract}
Neurons in the mammalian primary visual cortex are selective along multiple stimulus dimensions, including retinal position, spatial frequency, and orientation. Neurons tuned to different stimulus features but the same retinal position are grouped into retinotopic arrays of hypercolumns. This paper describes a neuromorphic implementation of orientation hypercolumns, which consists of a single silicon retina feeding multiple chips, each of which contains an array of neurons tuned to the same orientation and spatial frequency, but different retinal locations. All chips operate in continuous time, and communicate with each other using spikes transmitted by the address-event representation protocol. This system is modular in the sense that orientation coverage can be increased simply by adding more chips, and expandable in the sense that its output can be used to construct neurons tuned to other stimulus dimensions. We present measured results from the system, demonstrating neuronal selectivity along position, spatial frequency and orientation. We also demonstrate that the system supports recurrent feedback between neurons within one hypercolumn, even though they reside on different chips. The measured results from the system are in excellent concordance with theoretical predictions.
\end{abstract}

Index Terms-Address-event representation (AER), Gabor filter, image processing, mixed analog-digital integrated circuits, neural chips, neuromorphic engineering, visual cortex.

\section{INTRODUCTION}

$\mathbf{N}$ EUROMORPHIC engineering is the design and construction of systems that replicate the capabilities of biological systems, as well as their advantages, such as robustness and power efficiency by mimicking both functional and structural characteristics of biological systems [1].

We describe here a neuromorphic multichip implementation of orientation hypercolumns in the mammalian primary visual cortex (V1). In their "ice-cube" model, illustrated in Fig. 1(a), Hubel and Wiesel suggested that the visual cortex can be thought of as a two-dimensional (2-D) sheet with limited extent in depth [4]. At any point on this sheet, all of the neurons are tuned to the same orientation and location regardless of depth. An orientation tuned neuron's response to a bar is strongest when it is located at a preferred retinal location and with a preferred orientation, and weakens as the bar is moved or

Manuscript received April 8, 2004; revised September 22, 2004. This work was supported by the Hong Kong Research Grants Council under Grant HKUST6218/01E and by the National Science Foundation under CAREER Grant ECS00-93851. This paper was recommended by Associate Editor G. Cauwenberghs.

T. Y. W. Choi and B. E. Shi are with the Department of Electrical and Electronic Engineering, Hong Kong University of Science and Technology, Hong Kong (e-mail: eebert@ee.ust.hk).

P. Merolla, J. Arthur, and K. Boahen are with the Department of Bioengineering, University of Pennsylvania, Philadelphia, PA 19104-6392 USA.

Digital Object Identifier 10.1109/TCSI.2005.849136

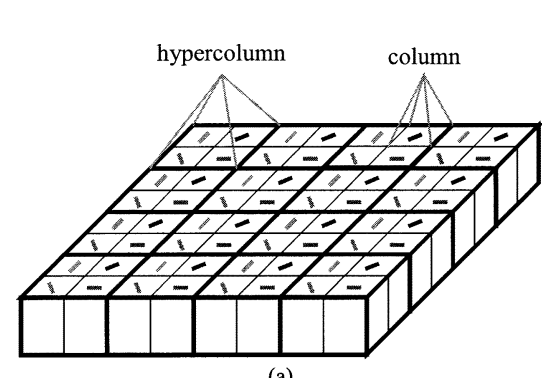

(a)

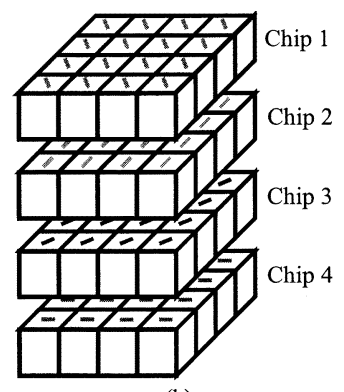

(b)

Fig. 1. (a) The "ice-cube" model of the visual cortex. Hypercolumns are divided by thick lines and subdivided by thin lines into columns of neurons tuned to different orientations as indicated by the oriented bars. (b) The multichip architecture used to implement the ice-cube model. Each layer represents one chip containing neurons tuned to the same orientation but different retinal positions. Vertically aligned neurons receive inputs from the same retinal locations.

rotated. Neurons tuned to the same orientation and retinal location are grouped into columns, which are depicted as cubes in Fig. 1(a). Neighboring columns that serve the same retinal location, but with different preferred orientations, are grouped into hypercolumns. Bold lines in Fig. 1(a) represent divisions between hypercolumns. These hypercolumns are arranged retinotopically, with neighboring hypercolumns serving neighboring retinal locations.

This work is an initial step in the construction of a neuromorphic system containing retinotopic arrays of continuous-time spiking silicon neurons exhibiting the same multidimensional stimulus selectivity observed in visual cortical neurons. Neurons in $\mathrm{V} 1$ are selective along the dimensions of retinal position, spatial frequency, temporal frequency, color, orientation, direction of motion, and binocular disparity [2]. Our goals in constructing this system are both to investigate hypotheses about the way biological system fuse information from different visual cues into a coherent perception of the environment, and to build biologically inspired systems for artificial perception. Our system operates in continuous-time to facilitate the incorporation of feedback interconnections between neurons, which appear to be critical for perception [3].

We have started by constructing orientation-tuned neurons because orientation selectivity is one of the predominant distinguishing characteristics of neurons in V1 [4]. Due to the processing by the retina, the input to cortex is already selective along the dimensions of retinal position, spatial frequency, temporal frequency, and color. In addition, selectivity along other stimulus dimensions commonly associated with V1, such as direction of motion and binocular disparity, can be obtained by combining the outputs of orientation-tuned neurons [5]-[7]. 
Our system, illustrated in Fig. 1(b), consists of a number of silicon chips. Each chip, called a Gabor chip, contains a 2-D array of silicon neurons, which are arranged retinotopically. However, unlike the biological cortex, all of the neurons in one chip have the same preferred orientation. To cover the orientation dimension, we use different chips, each containing neurons tuned to different orientations. Each chip in the system processes signals internally pixel-parallel and in continuous time using a combination of analog and asynchronous digital processing circuits. The chips interact with each other by spike-encoded inputs and outputs, which are communicated using the digital asynchronous address-event representation (AER) communication protocol [8]-[10].

We have previously described the circuit design of the Gabor chips used in this system, and characterized their operation in isolation with test input provided by a pattern generator [11]. This paper extends that work in several important directions. First, it describes experimental measurements of the output of the Gabor chips with optical input provided through a separate silicon retina chip. Second, it characterizes experimentally the operation of reconfigurable multichip systems of two to five chips linked by point-to-point AER routing circuits included in each chip. Third, it demonstrates experimentally that the AER protocol can be used effectively to implement continuous time feedback interactions between large arrays of spiking neurons on different chips.

This approach has several advantages over previous approaches to implementing orientation selectivity.

First, orientation resolution and spatial resolution can be controlled independently. Orientation resolution (the number of different orientation tuned neurons serving the same retinal location) can be increased by adding more Gabor chips. Spatial resolution (the number of retinal locations processed) can be increased by tiling more processing pixels in each chip. Since each neuron has the same orientation selectivity, its structure is identical to the other neurons on the chip, except for an offset due to the difference in the retinal position. In contrast, approaches that follow the ice-cube model by allocating different regions of silicon to different retinal locations and then further subdividing these regions for different orientations [12], [13], must trade off orientation resolution and spatial resolution. Although placing neurons tuned to different orientations on the same chip facilitates feedback interactions between them, we demonstrate here that our approach also supports such feedback interactions. However, the amount of interaction is limited by inter-chip bandwidth, which is slower and costlier in power than intra-chip bandwidth.

Second, the approach is expandable to include selectivity along more stimulus dimensions. We can combine the spatial filtering of these chips with temporal filtering to obtain velocity and direction tuned neurons [14] or combine the outputs of two chips processing left and right eye inputs to obtain binocular disparity tuned neurons [15]. Another approach to computing multiple feature maps is to time multiplex the same processing circuits and store the results of different maps in local pixel memories, as adopted by the Cellular Neural Network Universal Machine [16] or the computation on readout architecture [17]. However, multiplexing the computation of different feature maps in time makes it impossible to incorporate continuous-time recurrent interconnections between neurons with different stimulus selectivity.

Our approach is most similar to those reported by Serrano-Gotarredona et al. [18] and Venier et al. [19]. It differs primarily in the orientation selective receptive fields of the neurons, and in that we have been able to demonstrate feedback interactions between neurons tuned to different orientations experimentally. The approach proposed in [18] can implement only spatial filters with separable convolution kernels. Therefore, only horizontal or vertical orientation selectivity could be implemented. The system reported in [19] implements neurons with purely excitatory and even symmetric RF profiles. Thus, it cannot implement pairs of neurons with phase quadrature Gabor-like receptive fields.

The above discussion focused on architectures that are orientation selective, i.e., their responses vary according to the difference between the input orientation and a preferred orientation. Very large-scale integration (VLSI) architectures that measure feature orientation have also been proposed [20], [21]. However, we do not discuss them here, as the computations are quite different. For example, measurement assumes that each image point has a unique orientation. However, an orientation-selective architecture can support multiple orientation hypotheses at each point, e.g., the junction of two oriented edges.

In the following, Section II describes our multichip architecture. Section III gives measured results from our system demonstrating selectivity along three stimulus dimensions associated with V1: retinal position, spatial frequency, and orientation. We also demonstrate that our system supports recurrent interactions between neurons. Finally, Section IV concludes with summary and a description of the next steps in incorporating additional cortical functionality into this system.

\section{MultichiP ARCHITECTURE}

This section describes both a feedforward implementation of orientation hypercolumns, where the Gabor chips operate independently, and a feedback implementation, where the Gabor chips interact with each other.

Fig. 2(a) shows the block diagram of the feedforward implementation. The output of a silicon retina chip is fanned out to several Gabor chips. Fig. 2(b) shows a photograph of the feedforward system. Each Gabor chip is mounted on a separate printed circuit board, which also holds the circuits required to bias it. Each Gabor board dissipates $44 \mathrm{~mW}$ at $3 \mathrm{Kspike} / \mathrm{s}$. Most $(40 \mathrm{~mW}=90 \%)$ of the power consumption on the board is due to circuits that supply constant bias voltages to the chip and that power light emitting diodes (LEDs) that are used as status indicators.

The silicon retina, which is described in [22], [23], contains a $60 \times 96$ array of phototransistors and processing circuits that generate spike outputs that mimic the responses of ON-sustained and OFF-sustained retinal ganglion cells at a $30 \times 48$ array of retinal positions. ON and OFF neurons encode positive and negative contrasts relative to the local background intensity. Sustained retinal ganglion cells respond to inputs that vary slowly in time. 


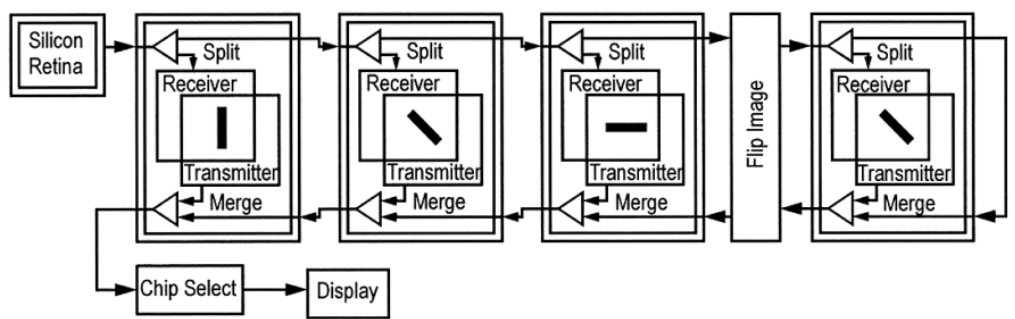

(a)

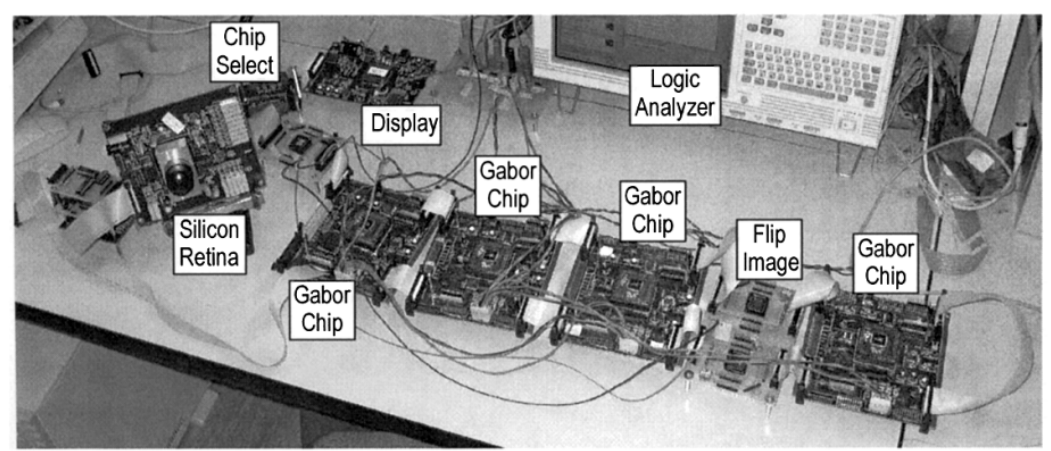

(b)

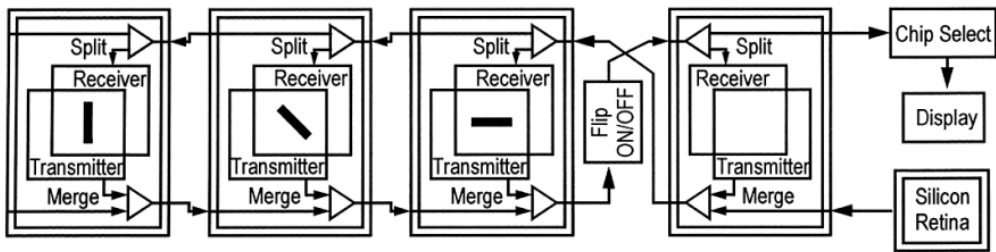

(c)

Fig. 2. (a) Feedforward implementation of orientation selective hypercolumns. Each box with the double line border represents a chip containing a retinotopic array of neurons. Gabor chips are represented by the larger boxes with the dark bar indicating the tuned orientation of the neurons on the chip. Boxes with single line borders indicate circuits that manipulate AER encoded spike trains. The "flip image" circuit remaps spike addresses to flip the input and output images of the fourth chip horizontally, resulting in neurons tuned to $135^{\circ}$. The chip select circuits pass only spikes originating from a desired chip. (b) Photo of the feedforward implementation. The silicon retina and Gabor chips are mounted on separate printed circuit boards with biasing circuits. The display board connects to a VGA monitor (not shown) for visualizing the chip outputs. The logic analyzer collects spikes for analysis. (c) Feedback implementation. The "flip ON/OFF" circuit inverts the image passing through it by mapping ON spikes to OFF spikes and vice versa. The missing bar in the right-most chip indicates that its spatial filtering is disabled.

Each Gabor chip can process ON and OFF spike input from a $32 \times 64$ array of retinal positions [11]. Each retinal position has four neurons associated with it. Each of those neurons computes a weighted sum of the spike rates from the ON and OFF ganglion cells in a small neighborhood of that retinal position, half-wave rectifies it, and encodes the result as an output spike rate. We refer to the weighting function used in the sum as the neurons receptive field (RF) profile. The four neurons are denoted by EVEN-ON, EVEN-OFF, ODD-ON, and ODD-OFF, and differ according to their RF symmetry (EVEN-ODD) and polarity (ON-OFF). The ON and OFF neurons encode the positive and negative half-wave rectified sums.

The RF symmetry with respect to the origin is determined by a phase parameter $\phi$. Each neuron's RF profile is a Gabor-like function given by

$$
g(x, y)=f(x, y) \cos \left(\Omega_{x} x+\Omega_{y} y+\phi\right)
$$

where $x$ and $y$ denote the horizontal and vertical distance from the neuron's position. The parameters $\Omega_{x}, \Omega_{y}$, and $\phi$ are real valued constants. Because the function $f(x, y)$ (described below) is even symmetric with respect to the origin, the RF is EVEN symmetric if $\phi=0$ and ODD symmetric if $\phi=-\pi / 2$.
When presented with a sine wave grating, the neurons will respond maximally to a grating with spatial frequency $\Omega=$ $\sqrt{\Omega_{x}^{2}+\Omega_{y}^{2}}$, orientation $\theta=\operatorname{atan}\left(\Omega_{y} / \Omega_{x}\right)$ and phase offset $\phi$. Vertical orientations correspond to $\theta=0$. The spatial frequency bandwidth is determined by the function $f(x, y)$, whose Fourier transform is given by

$$
\begin{aligned}
F\left(\omega_{x}, \omega_{y}\right) & =\frac{H_{\Omega}}{1+\frac{2-2 \cos \left(\omega_{x}\right)}{\left(\Delta \Omega_{x}\right)^{2}}+\frac{2-2 \cos \left(\omega_{y}\right)}{\left(\Delta \Omega_{y}\right)^{2}}} \\
& \approx \frac{H_{\Omega}}{1+\frac{\omega_{x}^{2}}{\left(\Delta \Omega_{x}\right)^{2}}+\frac{\omega_{y}^{2}}{\left(\Delta \Omega_{y}\right)^{2}}}
\end{aligned}
$$

where $H_{\Omega}, \Delta \Omega_{x}$, and $\Delta \Omega_{y}$ are positive constants. The parameter $H_{\Omega}$ controls the gain of the neuron. The parameters $\Delta \Omega_{x}$ and $\Delta \Omega_{y}$ control the spatial frequency bandwidth in the $x$ and $y$ directions. Although we do not have an exact closed form expression for $f(x, y)$, we know that it decays with distance from the origin and that the decay can be approximated by a zeroth order Bessel function of the second kind [24]. The parameters, $\Omega_{x}, \Omega_{y}, \Delta \Omega_{x}, \Delta \Omega_{y}$, and $H_{\Omega}$, are controllable via external bias voltages applied to the chip.

The neurons in our system capture many of the important characteristics of orientation tuned cortical neurons. The model 
of linear filtering followed by nonlinearity has been shown to account for the responses of a large proportion of V1 neurons [25]-[27]. The receptive field profile (1) has the same form as the Gabor functions commonly used to model orientation selective cortical neurons [28], [29], except that the modulating function $f(x, y)$ is not Gaussian. Pairs of EVEN and ODD neurons are critical in energy models of cortical function, and have been observed in cortex [30].

In contrast to conventional digital image processing systems where array readout is synchronized according to a pre-defined frame rate, output spike activity in our system is asynchronous. Each neuron in the array determines the time of its next spike output based upon the array's input and its last spike time. Neurons operate in continuous time and are free to generate a spike at any time. There is no clock synchronizing the activity in the array or its readout. Because the output activity depends upon the input activity, there is no well defined "frame" during which all neurons are read out.

We use the AER protocol [8]-[10] to communicate spike activity between chips. An AER link consists of a transmitter and a receiver connected by asynchronous digital lines. The transmitter signals that a neuron has spiked by sending an address event: a sequence of binary words identifying the spatial location (address) of the spiking neuron. Each spike is specified by three words that identify the chip, row and column that the spiking neuron resides in. The spike time is not encoded explicitly, but is taken to be the time at which the address event appears on the link. Simultaneous spikes from neurons in the same row and the same chip are transmitted in a single burst, which consists of the chip address, the row address, followed by the column addresses of the neurons that spiked. Simultaneous spikes from different rows are sequenced by arbitration. Since link bandwidth is allocated to the most active neurons, AER is more efficient than scanning when spike activity is sparse [31]. The Appendix describes the word-serial protocol we use, as well as the way in which the four types of neurons are addressed.

Since all spikes from one chip are transmitted via the same digital link, the total spike rate in the array is limited by the time which it takes to transmit one address event, which we measured to be $T_{\text {cyc }}=357 \mathrm{~ns}$. Subsequent events encoded in the same burst require only $T_{\mathrm{bst}}=106 \mathrm{~ns}$. For low loads where each burst contains only one address event, the link capacity is $1 / T_{\text {cyc }}=2.8$ million spikes per second. The link capacity in burst-mode is $1 / T_{\mathrm{bst}}=9.4$ million spikes per second. This burst rate does not indicate the true performance of the inter-chip links, as latches were interspersed between chips to correct for a design error in the communication protocol circuits.

Our architecture routes spikes between chips using point-topoint links, rather than a global bus connecting a number of transmitters and receivers. This avoids the need for complex circuits that control bus access and perform routing. Instead, we use two basic routing circuits, the split and the merge, which are included in each Gabor chip, and determine spike routing by the way that we link the chips together. Using this approach, routing circuit complexity expands automatically to accommodate the number of chips in the system. In addition, unique chip addresses are generated and updated automatically by the split and merge circuits, so that we can distinguish spikes from different chips in one AER address stream. The implementation of this network architecture will be described in detail in a forthcoming publication.

The split circuit makes two copies of the AER events appearing at its input. One copy is sent into the neuron array through a decoder that reads the originating address of the each spike and sends a spike to the neuron with the same row and column address, irrespective of the chip address. The other copy has its chip address incremented by one and is sent off chip via a transmitter.

Our system uses the split circuit for signal fan out. By daisy chaining the Gabor chips by connecting the split output of one with the split input of the next, we can distribute the same silicon retina output to all chips.

The merge circuit combines the address events at its input with address events generated by the neuron array and sends them off chip via a transmitter. Collisions between events at the input and the neuron array are handled by arbitration. Events coming from the neuron array are assigned a chip address of zero. Events coming from the input have their chip address incremented by one.

In our system, we use the merge circuits to collect the activity from all of the chips in the system, by daisy chaining the merge output of one chip with the merge input of the next. The merge output of each chip encodes all of the spike activity in the chips up to that point in the chain. Because it increments the chip address of input events, the merge circuit enables us to distinguish spikes originating in different chips. The "chip select" circuit is a filter that passes only spikes from a desired chip.

To minimize pixel size, we designed the Gabor chips so that they can only be tuned to orientations between $0^{\circ}$ and $90^{\circ}$. However, the system can contain neurons tuned to orientations greater than $90^{\circ}$, since the AER protocol makes it easy to flip images horizontally and/or vertically before and after processing. The Appendix describes the design of the "Flip Image" circuit.

Fig. 2(c) shows the block diagram of the feedback implementation of orientation hypercolumns. Each neuron on each Gabor chip is driven by a residual signal, which is the difference between the input from the silicon retina at its corresponding retinal location and the sum across orientation of the outputs of all the Gabor neurons serving the same retinal location with the same polarity and RF symmetry. The feedback system uses the merge and split circuits as well as a "combination chip" to compute the residual signal. The system first computes the inverted sum of the outputs of the Gabor chips, and then sums the input from the silicon retina with the inverted sum to compute the difference.

To compute the inverted sum across orientation, the system first collects the output spike activity from all of the Gabor chips at the input to the "flip ON/OFF" circuit by cascading the Gabor chips' merge circuits as in the feedforward system. The "flip ON/OFF" circuit, whose design is described in the Appendix, inverts the polarity of the Gabor chips' outputs by mapping ON spikes to OFF spikes and vice versa. The output of the "flip ON/OFF" circuit is fed to the split input of the combination chip, which sums the spike activity. The combination chip is simply 
a Gabor chip with its spatial filtering disabled. Its neurons integrate spikes corresponding to the same retinal location, polarity and symmetry but irrespective of orientation, since the decoder sends all spikes received at the split input into the neuron array irrespective of the chip address. For each retinal position and RF symmetry, the output spike rates of the ON and OFF neurons are approximately proportional to the half wave rectified differences between their input spike rates.

The combination chip's merge circuit combines the input spike activity from the silicon retina with the output of the combination chip, which represents the inverted sum of the Gabor chips' outputs. These spikes are distributed to each of the Gabor chips by cascading the split circuits as in the feedforward system. Although spikes from the silicon retina and the combination chip can be distinguished by their chip addresses, they are summed by the Gabor chips since the decoder ignores the chip addresses when routing spikes from the split input into the neuron array.

Intuitively, the net effect of this feedback connectivity is that the system automatically adjusts the Gabor chip outputs so that their sum at each location best fits the input from the silicon retina at that location. The residual measures the quality of the fit. If the residual has a large component near the tuned orientation of Gabor chip, that chip increases its response since the residual signal is fed into its input. The interaction between neurons tuned to different orientations is inhibitory, since if the neurons tuned to one orientation are providing a good fit to the input, they decrease the input to neurons tuned to other orientations. The extensive feedback interconnectivity in this system might raise concern about its stability. However, we have not observed any unstable or oscillatory behavior in our experiments. In addition, it is possible to prove that a similar system where the spiking interactions are replaced by graded interactions is stable, since its dynamics can be characterized as gradient descent on a Lyapunov function similar to that presented in [33].

\section{EXPERIMENTAL RESULTS}

This section describes experimental measurements from both the feedforward and feedback implementations of the orientation selective hypercolumns. We demonstrate the position, spatial frequency, and orientation selectivity of the neurons using the feedforward system. In the feedback system, we focus on the effect of the competitive interactions on the orientation tuning, which is the primary difference in the response characteristics of neurons in the two systems.

\section{A. Feedforward System}

We tuned the four Gabor-type chips in the feedforward system to similar spatial frequencies and bandwidths, but different orientations $0^{\circ}, 45^{\circ}, 90^{\circ}$, and $135^{\circ}$, with $135^{\circ}$ achieved by tuning the chip to $45^{\circ}$ and then flipping the input and output images horizontally. In total, this system contains 32768 orientation tuned neurons tuned to four orientations, two spatial phases, two polarities (ON/OFF) and $32 \times 64$ retinal positions. Including the retina, the system contains 35648 spiking neurons. We estimated the tuning parameters $\Omega_{x}, \Omega_{y}, \Delta \Omega_{x}$, and $\Delta \Omega_{y}$ for each chip by applying a spatial impulse input to the
TABLE I

TUNING PARAMETERS EstimATED Via IMPULSE RESPONSE FITTING

\begin{tabular}{c|c|c|c|c}
\hline & $0^{\circ}$ & $45^{\circ}$ & $90^{\circ}$ & $135^{\circ}$ \\
\hline$\Omega_{\mathrm{x}}$ & 0.59 & 0.41 & 0.03 & -0.42 \\
\hline$\Omega_{\mathrm{y}}$ & 0.01 & 0.42 & 0.55 & 0.43 \\
\hline$\Delta \Omega_{\mathrm{x}}$ & 0.56 & 0.42 & 0.54 & 0.41 \\
\hline$\Delta \Omega_{\mathrm{y}}$ & 0.58 & 0.46 & 0.56 & 0.42 \\
\hline$\Omega=\sqrt{\Omega_{\mathrm{x}}^{2}+\Omega_{\mathrm{y}}^{2}}$ & 0.59 & 0.59 & 0.55 & 0.60 \\
\hline$\theta=\operatorname{atan}\left(\Omega_{\mathrm{y}} / \Omega_{\mathrm{x}}\right)$ & $0.7^{\circ}$ & $45.4^{\circ}$ & $87.3^{\circ}$ & $134.6^{\circ}$ \\
\hline
\end{tabular}

center pixel using a pattern generator and measuring the output across the array, and finding the parameters that minimized the squared error between the measured and theoretically predicted responses (Table I). This estimation assumes the tuning parameters of the neurons are identical. In fact, the tuning parameters vary from neuron to neuron due to transistor mismatch, with different parameters exhibiting different amounts of variability [15].

Fig. 3 shows the responses of the neurons in response to a dark ring on a bright background. Neurons from different chips respond to parts of the ring, depending on where their tuned orientation match the ring's. The ODD neurons are more sharply tuned in orientation than the EVEN neurons. We used a $4 \mathrm{~mm}$ lens to focus images onto the surface of the silicon retina, and presented visual stimuli to the system using an liquid crystal display (LCD) monitor placed $25 \mathrm{~cm}$ away. Transistor mismatch adds variation in the neural responses across position, due to changes in the gain, tuning, and background firing rates of the neurons [11], [15].

To evaluate spatial frequency and orientation tuning of the neurons, we presented the system with Gabor patches with a constant circularly symmetric Gaussian envelope but varying orientation and spatial frequency. To avoid edge effects, we ensured that the Gabor patch was confined within the field of view of the retina by choosing the standard deviation of the Gaussian to span $5.4^{\circ}$ of visual angle, which corresponded to the spacing between the receptive field centers of 8.4 SUSTAINED cells in the silicon retina. Fig. 4 shows images of Gabor patches plotted with examples of the parameters used.

To evaluate the spatial frequency tuning of the system, we varied the spatial frequency of the Gabor patch while keeping the orientation constant at $90^{\circ}$ and monitored the response of the array tuned to $90^{\circ}$. By measuring the total spike rate from all neurons irrespective of retinal position, RF symmetry and polarity, we eliminate the dependency of the response on the spatial phase of the stimulus, leaving only the dependency on the spatial frequency. For each spatial frequency, we measured the spike times of 600 spikes from the chip to compute the spike rate. Fig. 5 shows the population response of the retinal neurons and the orientation-tuned neurons plotted versus spatial frequency. The spatial frequency that gave the greatest response was 0.6 radians/pixel, which agrees with the results from the impulse response fitting. This spatial frequency corresponds to 0.16 cycles per degree of visual angle. Much of the spatial 


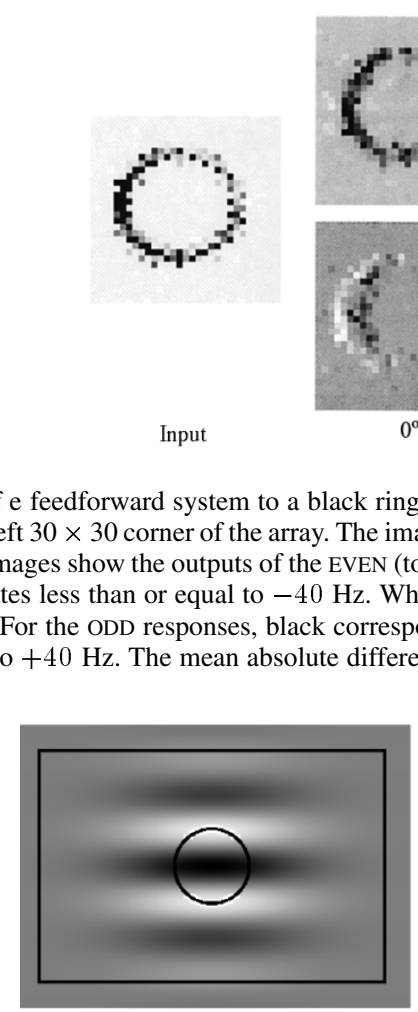

(a)
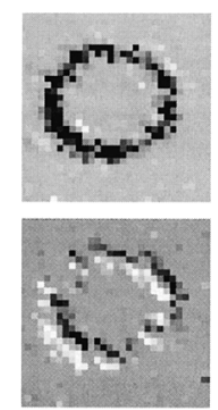

$45^{\circ}$
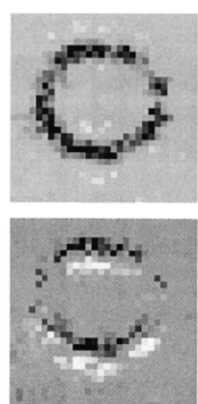

$90^{\circ}$
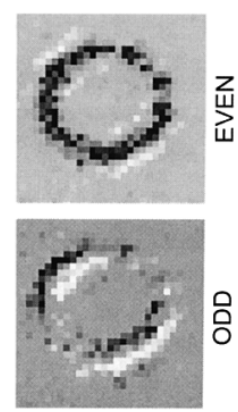

$135^{\circ}$

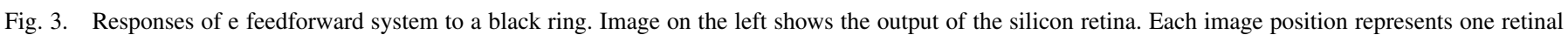

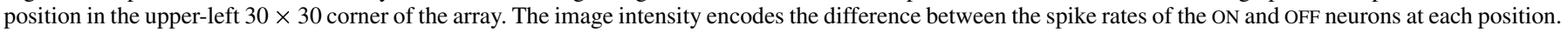

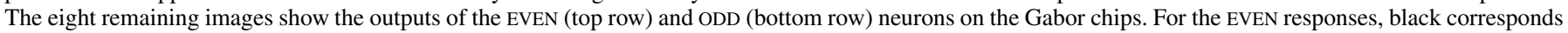

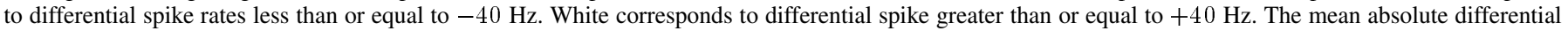

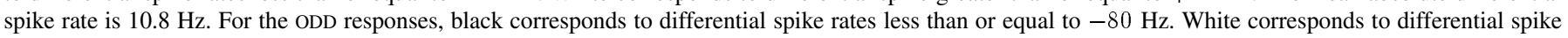
greater than or equal to $+40 \mathrm{~Hz}$. The mean absolute differential spike rate is $4.4 \mathrm{~Hz}$.

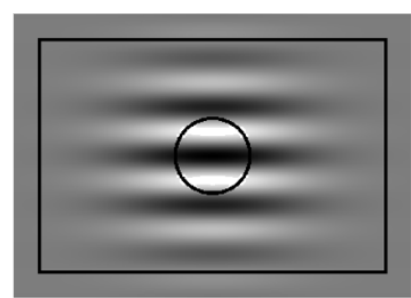

(b)

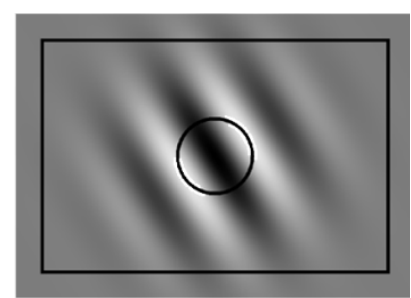

(c)

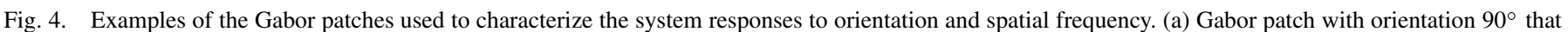

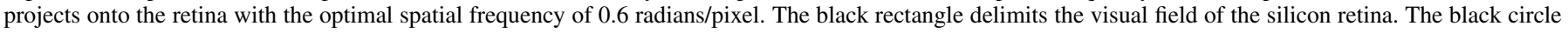

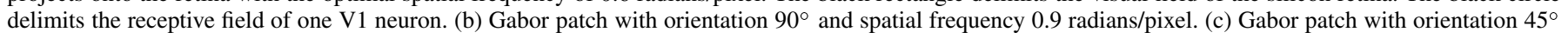
spatial frequency 0.6 radians/pixel.

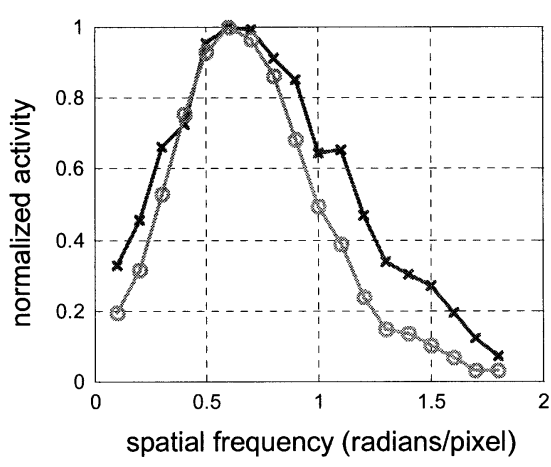

Fig. 5. Spatial frequency tuning of the neurons in the retina (x) and the Gabor chip (o). To facilitate comparison, both curves are normalized by the peak population spike rate.

frequency selectivity is due to bandpass filtering by the retina, which is tuned to the same spatial frequencies as the orientation tuned neurons, but is not orientation selective.

To characterize the orientation tuning of the neurons, we fixed the spatial frequency of the Gabor patches at 0.6 radians/pixel, which yielded the maximum response in the previous experiment, and varied the orientations from 0 to $180^{\circ}$ in steps of $11.25^{\circ}$. Because we used stationary gratings, orientations greater than or equal to $180^{\circ}$ are equivalent to orientations less than $180^{\circ}$. Fig. 6 shows the measured population responses from the EVEN and ODD neurons, which were obtained by summing the responses from all neurons with the same RF symmetry on each chip, irrespective of retinal position or polarity. The polar plots show that neurons on different chips are tuned to respond maximally to different orientations, and that the ODD neurons are more sharply tuned in orientation than the EVEN neurons.

Fig. 6 also compares the measured data with the theoretical predictions. The equations used to generate the theoretical fits are given in the Appendix. We assumed that the spatial frequency of the input sine wave gratings and the spatial frequency tuning $\Omega$ of all the chips was 0.6 radians/pixel, and that the neurons were tuned to orientations $0^{\circ}, 45^{\circ}, 90^{\circ}$, and $135^{\circ}$ exactly. We also assumed that $\Delta \Omega_{x}=\Delta \Omega_{y}$ and that they were identical in all chips. We estimated the common value to be 0.61 radians per pixel by a least squares fit to the measured data. This estimated value is slightly larger than the value predicted by the impulse response fits, primarily because the Gabor patches used in the experimental characterization are spatially localized, and therefore have broader spatial frequency content than the sine wave grating assumed in the theoretical analysis. Although it is possible to predict the responses to Gabor patches numerically, we do not do so here because the assumption of sine wave grating inputs matches the measured responses quite closely and gives a convenient closed form expression. Other contributing factors include the fact that the impulse response data was measured by stimulating a single pixel, which is not an accurate measure of the average tuning across the neurons in the array due to transistor mismatch, as well as the additional spatial filtering performed by the silicon retina. 


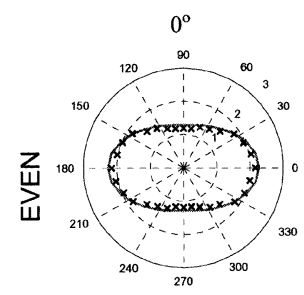

$\mathrm{PO}=-0.2^{\circ}$

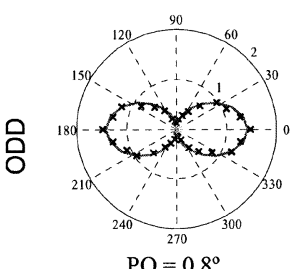

$\mathrm{PO}=0.8^{\circ}$

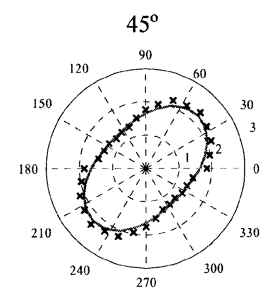

$\mathrm{PO}=44.8^{\circ}$

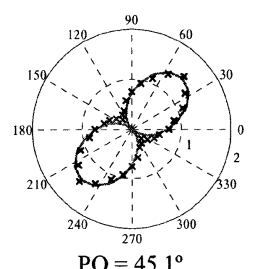

$\mathrm{PO}=45.1^{\circ}$
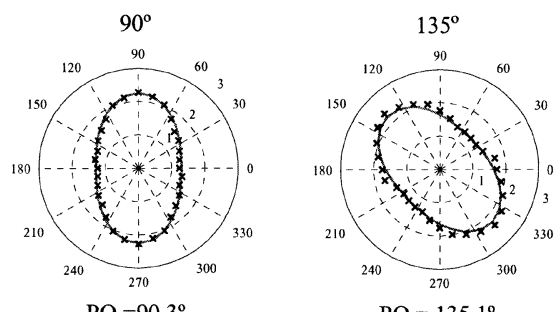

$\mathrm{PO}=135.1^{\circ}$

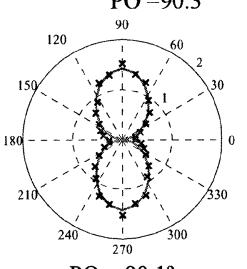

$\mathrm{PO}=90.1^{\circ}$

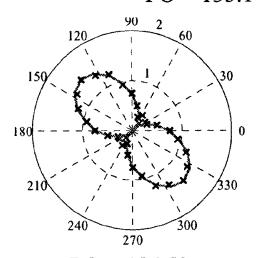

$\mathrm{PO}=134.8^{\circ}$

Fig. 6. Orientation tuning of the feedforward system. Polar plots show the total spike rate from all neurons with the same RF symmetry from one chip. For each point, the angle represents the input orientation and the distance from the origin represents the total spike rate in kilohertz minus the background spike rate measured in response to a blank screen. Top row shows the response of the EVEN neurons. Bottom row shows the response of the ODD neurons. Each column shows responses from a different chip. Discrete data points $x$ indicate measured data. Solid lines show the theoretical fit. Labels at the top of each column indicate the target uncoupled tuning. Label below each graph indicates the preferred orientation computed from the measured data.

TABLE II

SYSTEM CHARACTERISTICS

\begin{tabular}{c|c|c|c|c}
\hline & $\begin{array}{c}\text { \# of Gabor } \\
\text { Boards }\end{array}$ & $\begin{array}{c}\text { \# of } \\
\text { Neurons }\end{array}$ & $\begin{array}{c}\text { GoF(\%) } \\
\text { EVEN }\end{array}$ & $\begin{array}{c}\text { GoF (\%) } \\
\text { ODD }\end{array}$ \\
\hline Feedforward system & 4 & 35,648 & 99.4 & 88.8 \\
\hline Self coupled feedback system & 2 & 19,264 & 93.1 & 84.8 \\
\hline 2 orientation feedback system & 3 & 27,456 & 93.7 & 81.6 \\
\hline 3 orientation feedback system & 4 & 35,648 & 54.2 & 75.7 \\
\hline \hline Gabor board power dissipation & \multicolumn{4}{|c}{ 44mW@3Kspikes/second } \\
\hline AER cycle/burst time & \multicolumn{5}{|c}{ 346ns/106ns } \\
\hline
\end{tabular}

For each RF symmetry, we evaluated the quality of the theoretical fits using a goodness of fit index defined by

$$
G o F=\left(1-\sqrt{\frac{\Sigma_{k}\left(o_{k}-p_{k}\right)^{2}}{\sum_{k} o_{k}^{2}}}\right) \times 100 \%
$$

where $o_{k}$ is the observed response (in spikes per second) and $p_{k}$ is the theoretically predicted response for measurement $k$. The summation is taken over all input orientations and over all chips. A perfect fit gives $G o F=100 \%$.

The goodness of fit indexes for all of the systems are given in Table II, which summarizes the system characteristics. In general, the fit between the predicted and measured responses from the ODD neurons is worse than the fit for the EVEN neurons. The degradation is largely due to the fact that the theory predicts zero response to orthogonal orientations, which is not the case for the measurements.

We define the preferred orientation (PO) of an orientation tuning curve to be $0.5 \angle R$ where $R$ is the resultant [34]

$$
R=\frac{\Sigma_{k} o_{k} e^{2 \theta_{k}}}{\Sigma_{k} o_{k}}
$$

and $o_{k}$ is the response to the input orientation $\theta_{k}$. We double $\theta_{k}$ in the complex exponential and subsequently halve the angle of $R$ to take into account the fact that orientations that differ by $180^{\circ}$ are equivalent. The preferred orientation computed from the measured data and theoretical fit, which are given in Fig. 6, are in good concordance with the target tuning.

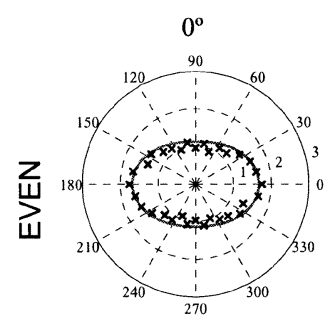

$\mathrm{PO}=0.2^{\circ}$

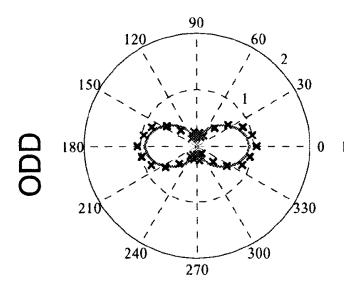

$\mathrm{PO}=1.2^{\circ}$

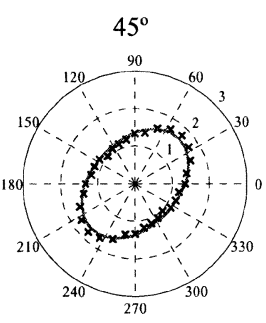

$\mathrm{PO}=43.9^{\circ}$

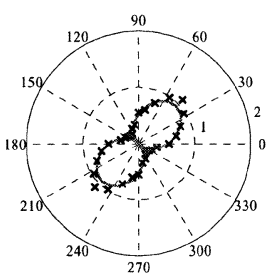

$\mathrm{PO}=44.8^{\circ}$

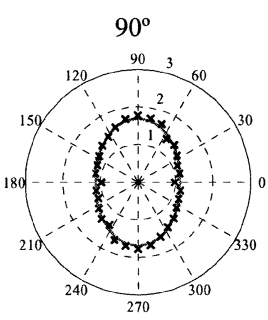

$\mathrm{PO}=89.8^{\circ}$

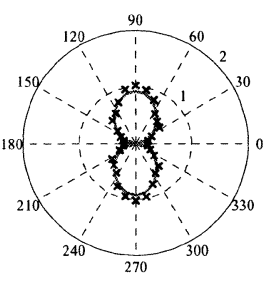

$\mathrm{PO}=89.0^{\circ}$
Fig. 7. Orientation tuning of the self-coupled system. Polar plots generated as in Fig. 6. Label below each graph indicates the preferred orientation computed from the measured data.

\section{B. Feedback System}

We characterized several different configurations of the feedback system, which varied in the number of Gabor chips coupled together. In the following, we refer to the responses of the neurons in the feedforward system as uncoupled, and the responses of the neurons in the feedback system as coupled. The simplest feedback system, which we refer to as self-coupled, contains one Gabor chip coupled back to itself through the combination chip. The next level of complexity couples two Gabor chips tuned to different orientations, where the inhibitory feedback shifts the orientation tuning curves for the two sets of neurons away from each other. Finally, we characterized a system with three Gabor chips. These experiments used the same bias voltages to set the uncoupled tuning parameters as used in the feedforward experiments.

We characterized the self-coupled system for the Gabor chips tuned to orientations of $0,45^{\circ}$, and $90^{\circ}$ with the same bias 

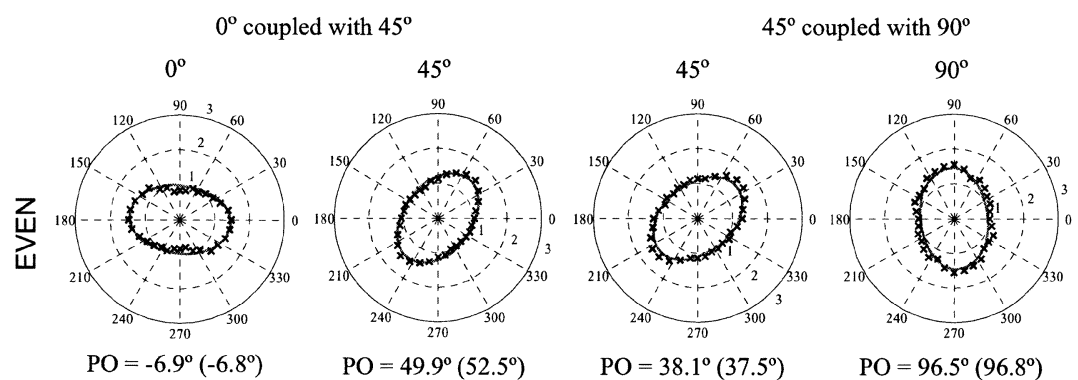

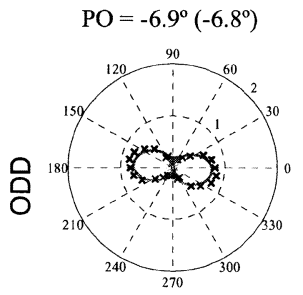

$\mathrm{PO}=-6.6^{\circ}\left(-6.9^{\circ}\right)$

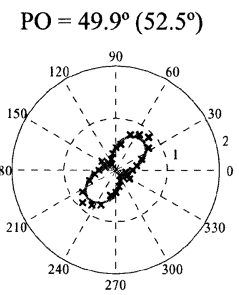

$\mathrm{PO}=49.5^{\circ}\left(51.7^{\circ}\right)$

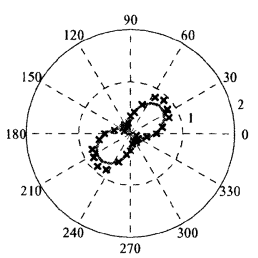

$\mathrm{PO}=39.4^{\circ}\left(38.3^{\circ}\right)$

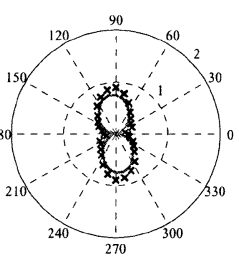

$\mathrm{PO}=95.1^{\circ}\left(96.9^{\circ}\right)$

Fig. 8. Orientation tuning with two coupled orientations. Polar plots generated as in Fig. 6. Label below each graph indicates the preferred orientation computed from the measured data and predicted theoretically (in parenthesis).

voltages as used in the feedforward system. Fig. 7 shows the polar plots of the measured response and theoretical fit. The experimental measurements were performed using the same protocol as for the feedforward system. The theoretical fit was obtained using the equations described in the Appendix, where we model the feedback as linear with a gain $\beta$, which we estimated to be 0.41 by a least squares fit to the experimental measurements. The remaining parameters were the same as used to fit the responses of the feedforward system.

The primary difference between the responses of the neurons in the feedforward and self-coupled system is a reduction in the responses of the neurons of the self-coupled system. In the selfcoupled system, each neurons output is inverted and fed back to its own input. Thus, each neuron inhibits itself, accounting for the reduced response. There is no significant change in the preferred orientation or the sharpness of the orientation tuning.

To characterize the two Gabor chip system, we fixed the uncoupled tuning of one Gabor chip at $45^{\circ}$, and coupled it with a second chip tuned to $0^{\circ}$ or $90^{\circ}$. We measured orientation tuning using the same protocol as our previous experiments. In the following, we refer to the chips and the neurons according to their uncoupled tunings. Fig. 8 shows the orientation tuning curves and preferred orientations obtained. The theoretical predictions are based upon the parameters estimated in our previous experiments, and therefore are true predictions of the effect of feedback.

Comparing the $45^{\circ}$ neurons in the two cases (the two center columns), we observe that their preferred orientations shift toward higher orientations when they are coupled with $0^{\circ}$ tuned neurons, and toward lower orientations when coupled with $90^{\circ}$ tuned neurons. Similarly, we observe that the preferred orientation of the $0^{\circ}$ neurons (the first column) shifts toward negative orientations. The preferred orientation of the $90^{\circ}$ neurons (the last column) shifts toward orientations greater than $90^{\circ}$.

When two sets of orientation-tuned neurons are coupled together, their tuning curves shift away from each other so that the coupled tuning curves are more separated than the uncoupled tuning curves. Intuitively, consider two neurons, labeled 1
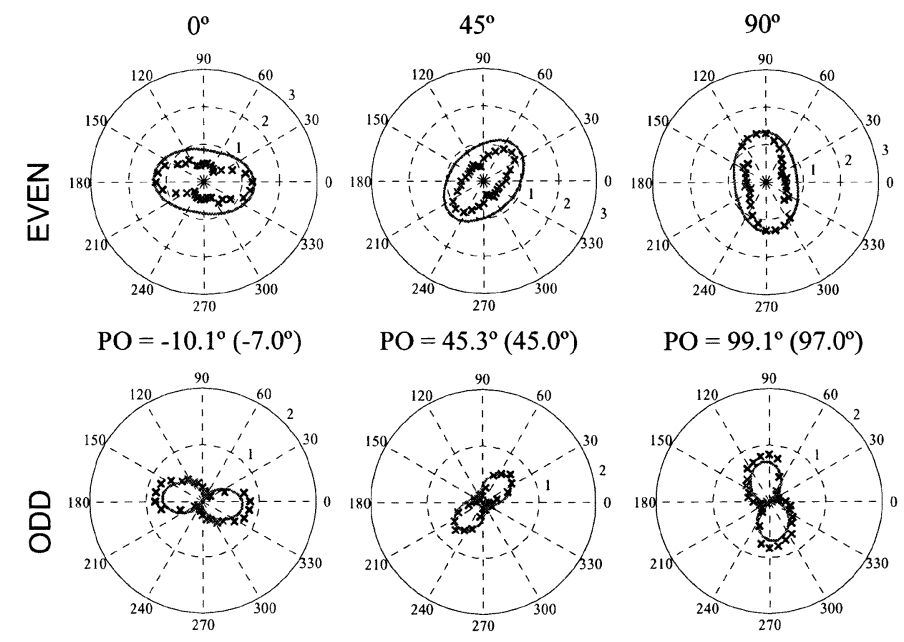

$\mathrm{PO}=45.3^{\circ}\left(45.0^{\circ}\right)$

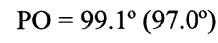

$\mathrm{PO}=-19.1^{\circ}\left(-11.4^{\circ}\right)$

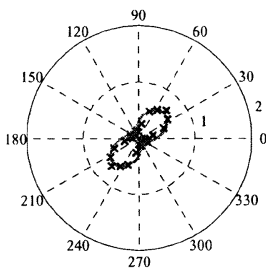

$\mathrm{PO}=45.2^{\circ}\left(45.0^{\circ}\right)$

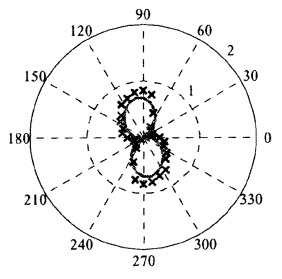

$\mathrm{PO}=109.0^{\circ}\left(101.4^{\circ}\right)$
Fig. 9. Orientation tuning with three coupled orientations, $0^{\circ}, 45^{\circ}$, and $90^{\circ}$. Polar plots generated as in Fig. 6. The label below each graph indicates the preferred orientation computed from the measured data and predicted theoretically (in parenthesis).

and 2, that respond to the same retinal region but different orientations. Feedback interactions will enhance the difference in orientation tuning because inhibition from Neuron 2 reduces the response of Neuron 1 to orientations that are near the preferred orientation of Neuron 2.

Fig. 9 shows the tuning curves and preferred orientations obtained when coupling three chips with uncoupled tunings of $0^{\circ} / 45^{\circ} / 90^{\circ}$. As in the previous experiment, the theoretical predictions are based upon the parameters identified in the feedforward and self-coupled experiments.

In this case, the prediction is not as good as the prediction with only two coupled orientations. In particular, the magnitude of the response is smaller for the EVEN neurons than predicted theoretically, resulting in a much poorer fit than observed for the other systems. It appears that inhibition by two competing orientations is greater than that predicted by the linear model in the Appendix. Because we have been unable to fit the responses 
accurately by increasing the strength of the feedback factor, we speculate that nonlinearities introduced by the spike-based communication between chips, e.g., in spike generation and leaky integration, may be responsible for the discrepancy between the theoretical predictions and the measured data.

The net shift in the tuning curve for the $45^{\circ}$ neurons is close to zero, since the shifts introduced by $0^{\circ}$ and $90^{\circ}$ neurons cancel. The tuning curves of the $0^{\circ}$ and $90^{\circ}$ neurons shift in the same direction as in the two Gabor chip system, but by a greater amount due to the increased inhibition on the same side of the tuned orientation.

\section{CONCLUSION}

Based upon the columnar organization of orientation selective neurons in the mammalian primary visual cortex (V1), we have constructed a silicon system consisting of retinotopic arrays of silicon neurons whose receptive field properties closely match those of orientation selective visual cortical neurons. The system uses a similar ON-OFF signal representation as used in biological neural systems to encode signals above and below a mean value.

We have built and characterized a feedforward system where the output of a silicon retina is fanned out to several Gabor chips that are tuned to different orientations, but which operate independently. Our measurements from the feedforward system demonstrate that the neurons in this system exhibits stimulus selectivity along three dimensions commonly associated with neurons in V1: retinal position, spatial frequency, and orientation.

We have also demonstrated that even though we implement neurons that normally reside within a single hypercolumn in the biological cortex on different chips, our system can still support feedback interactions between them. This is a critical feature of a neuromorphic model of cortex since much of the input to cortical cells comes from other cortical cells [35]. Our results show that a linear model can account for over $80 \%$ of the response of a feedback system consisting of two populations of neurons tuned to different orientations.

In our system, inhibitory feedback shifts the orientation tuning. In the biological cortex, similar shifts in orientation tuning due to changes in feedback can account for changes in neuronal response due to adaptation and learning [36]-[38].

Another hypothesized role for intracortical inhibition is to balance local recurrent cortical excitation, leading to orientation sharpening and contrast invariant tuning [35]. In our system, contrast invariant tuning is achieved in a different manner, via push-pull interactions between ON and OFF neurons similar to those proposed in [39]. However, although our current system does not exhibit any significant orientation sharpening, with the exception of the measured data from the even neurons in Fig. 9, the next generation of our system should be able to be configured to exhibit orientation sharpening. Our theoretical analysis indicates that the lack of orientation sharpening in our current system is primarily because each neuron inhibits itself, since the residual signal fed back to each neuron contains its own output. If we eliminate this inhibitory self feedback, the analysis in [33] predicts that the resulting system will exhibit sharpened orientation tuning. One reason it is difficult

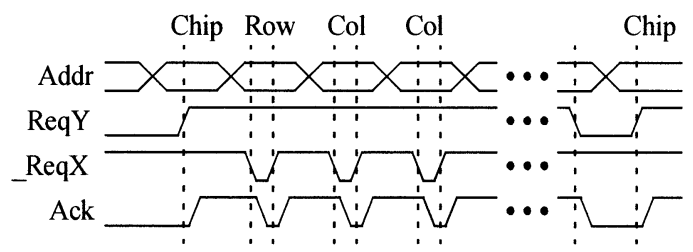

Fig. 10. Address protocol used by the Gabor-type filter chip. A chip and row address is followed by one or more column addresses, depending upon how many neurons in a given row of the chip spiked simultaneously. Since the protocol is asynchronous, the transmitter and receiver use handshaking signals to ensure addresses are communicated correctly. Two request signals, ReqY and $\sim$ ReqX, identify the start and end of each burst, and the location of each address within the burst. Transmitter raises ReqY to signal the validity of the chip address. Receiver acknowledges that it has read the chip address by raising the acknowledge Ack. The transmitter then lowers $\sim$ ReqX to signal the validity of the row and column addresses. Receiver acknowledges receipt by lowering Ack. Transmitter lowers ReqY to signal the end of the burst.

to implement this type of system using our current chips is that the decoder at the split input sends all spikes into the array irrespective of the chip address. However, our next generation of routing circuits will include the capability to decide whether or not to route spikes into the neuron array depending upon the chip address. This will facilitate the construction of a system that does not include inhibitory self-feedback.

\section{APPENDIX}

\section{A. Word-Serial AER Format}

The Gabor chips use the 7-bit word serial format for addresses illustrated in Fig. 10. Spikes are sent in sequences of addresses, called bursts. The first address identifies the chip, the second address identifies the row and the remaining addresses identify the columns containing neurons in the row that spiked. This "burst mode" improves efficiency when there is high activity in the array, by eliminating the time needed to send the same chip and row addresses for each column address. Because the AER protocol is asynchronous, we use handshaking signals to ensure addresses are received properly.

Within each Gabor neuron array, ON and OFF neurons are addressed in alternate columns, while EVEN and ODD neurons are addressed in alternate rows. Thus, the LSB of the column address identifies the neuron's polarity. The LSB of the row address identifies the receptive field symmetry. In the silicon retina, ON and OFF neurons are also addressed in alternate columns. SUSTAINED and TRANSIENT neurons are addressed in alternate rows. Thus, the SUSTAINED outputs of the retina map directly to the inputs of the EVEN neurons with the same polarity. In the feedforward system, the ODD neurons are driven indirectly through recurrent feedback interconnections with the EVEN neurons on each chip. In the feedback system, they are also driven by the ODD component of the residual signal. In this system, we disable the TRANSIENT retina outputs, which encode quickly varying image components.

\section{B. Spike Remapping and Filtering Circuits}

The "flip image" circuit can flip an image horizontally and/or vertically by remapping the row and/or column addresses within each burst. We generate a flipped row or column address by inverting all of the bits except for the LSB, which encodes the 


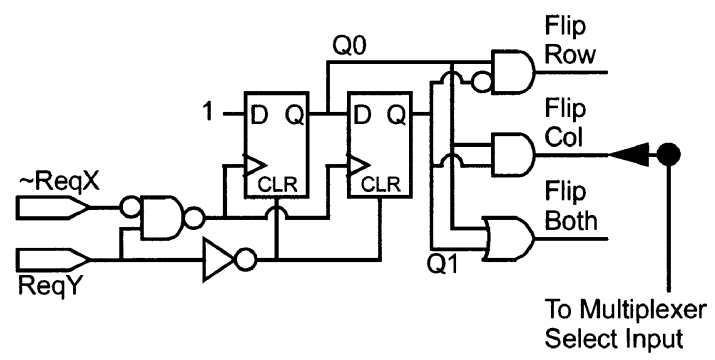

(a)

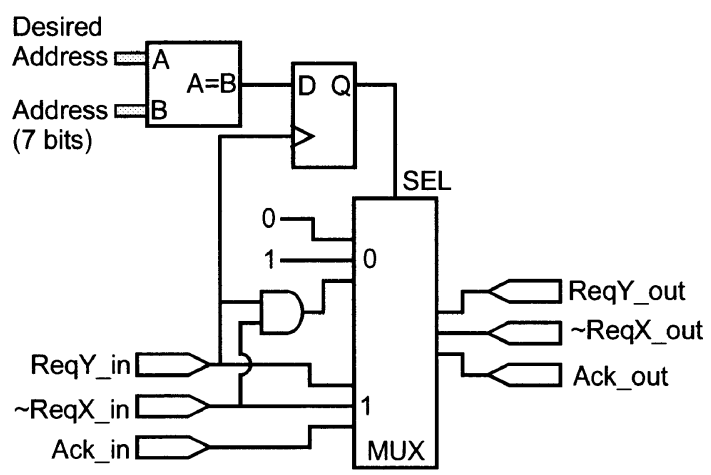

(b)

Fig. 11. (a) Multiplexer control for the "image flip" circuit. The shift register outputs are cleared when ReqY is low. Once ReqY goes high, signalling the presence of a burst, high bits are clocked into the shift register at every low transition of the $\sim \operatorname{ReqX}$. At the chip address, the outputs $(Q 0, Q 1)$ are $(0,0)$. For the row address, the outputs are $(1,0)$. For all of the column addresses, the outputs are $(1,1)$. Switch determines how the SELECT input of the multiplexer is controlled to flip the row and/or column address. (b) Schematic of a chip select block. When ReqY_in goes high, the select input to the multiplexer is latched high or low, depending on whether the chip address matches the desired chip address. If the chip address matches, then the Req $\mathrm{Y}$ in, $\sim \operatorname{ReqX}$ in, and Ack in signals are passed to ReqY_out, $\sim \operatorname{ReqX}$ _out, and Ack_out. Otherwise, the ReqY_out signal stays low, the $\sim$ ReqX_out signal stays high and the $\mathrm{Req} Y$ in and $\sim \operatorname{ReqX}$ in signals are used to generate the Ack_out signal to complete the communication cycle.

symmetry or polarity. A multiplexer sends either the original or the flipped address. The logic to generate the multiplexer select signal is shown in the schematic of Fig. 11(a). The "flip on/off" circuit is similar. It uses the column flip logic to select whether to send the original address, or an address with the LSB inverted. Both are implemented on Xilinx field programmable gate arrays (FPGAs).

The "chip select" circuit is placed between a transmitter and a receiver, and passes only bursts corresponding to a desired chip. If the chip address of a burst matches that of the desired chip, then the circuit is transparent to the ReqY, $\sim \operatorname{ReqX}$, and Ack signals. Otherwise, it blocks the ReqY and $\sim$ ReqX signals from the transmitter, and generates Ack signal locally. There is no need to block the address bits, since data on the address lines is ignored unless a request is detected. Fig. 11(b) gives the schematic of this circuit, which we implement on a Xilinx FPGA.

\section{Theoretical Fits to Neuronal Responses}

This section gives the expressions used to fit the tuning curves for the EVEN and ODD neurons measured in the feedforward and feedback systems. We derive the spatial transfer function of the orientation selective neurons in the feedback network by assuming the feedback interactions between neurons is linear. The analysis is similar to that in [33], but differs because the residual signal sent to each neuron in [33] does not include its own output.

Each Gabor chip contains four arrays of neurons labeled EVEN/ON $(e+)$, EVEN/OFF $(e-)$, ODD/ON $(o+)$ and ODD/OFF $(o-)$. For each spatial position $(m, n)$, let $u_{k}(m, n)$ denote the input spike rates of the four neurons $k \in\{e+, e-, o+, o-\}$. These differentially encode two real numbers $u_{e}(m, n)$ and $u_{o}(m, n)$, where $u_{e}(m, n)=u_{e+}(m, n)-u_{e-}(m, n)$ and similarly for $u_{o}(m, n)$. These two real numbers can be expressed as a single complex number $u(m, n)=u_{e}(m, n)+j u_{o}(m, n)$ where $j=\sqrt{-1}$. Similarly, the output spike rates $x_{k}(m, n)$ encode numbers $x_{e}(m, n), x_{o}(m, n)$ and $x(m, n)$. Internally, the chip performs spatial filtering on arrays of input currents to produce arrays of output currents. Assuming that the conversion from spike rate to current and vice versa is linear, the steady-state input and output images are related by the equations

$$
X(\vec{\omega})+D(\vec{\omega}) X(\vec{\omega})-H_{\Omega} U(\vec{\omega})=0
$$

where capital letters denote discrete spatial Fourier Transforms $\vec{\omega}=\left(\omega_{x}, \omega_{y}\right)$ denotes spatial frequency, and

$$
D(\vec{\omega})=\frac{2-2 \cos \left(\omega_{x}-\Omega_{x}\right)}{\left(\Delta \Omega_{x}\right)^{2}}+\frac{2-2 \cos \left(\omega_{y}-\Omega_{y}\right)}{\left(\Delta \Omega_{y}\right)^{2}}
$$

where $\Omega_{x}, \Omega_{y}, \Delta \Omega_{x}$, and $\Delta \Omega_{y}$ are tuning parameters, which are set by external bias voltages, and $H_{\Omega}$ is a gain factor that depends upon the tuning parameters and constants of proportionality introduced by the spike rate to current conversion and vice versa.

Solving (3), we obtain

$$
X(\vec{\omega})=H(\vec{\omega}) U(\vec{\omega})
$$

where

$$
H(\vec{\omega})=\frac{H_{\Omega}}{1+D(\vec{\omega})} .
$$

The transfer function achieves its maximum at $\vec{\omega}=\left(\Omega_{x}, \Omega_{y}\right)$ where $D(\vec{\omega})=0$ and drops by half at $\vec{\omega}=\left(\Omega_{x} \pm \Delta \Omega_{x}, \Omega_{y}\right)$ and $\vec{\omega}=\left(\Omega_{x}, \Omega_{y} \pm \Delta \Omega_{y}\right)$ where $D(\vec{\omega})=1$. By the Fourier shift theorem, the filter kernel has the form $h(m, n)=f(m, n) \exp \left(j \Omega_{x} m+j \Omega_{y} m\right)$ where $f(m, n)$ is the inverse Fourier transform of $H(\vec{\omega})$ when $\Omega_{x}=\Omega_{y}=0$, which is a real valued function that decays with distance from the origin. If we define $h_{e}$ and $h_{o}$ to be the real and imaginary parts of $h$, we have

$$
\begin{aligned}
& h_{e}(m, n)=f(m, n) \cos \left(\Omega_{x} x+\Omega_{y} y\right) \\
& h_{o}(m, n)=f(m, n) \cos \left(\Omega_{x} x+\Omega_{y} y-\pi / 2\right)
\end{aligned}
$$

which have Fourier transforms

$$
\begin{aligned}
& H_{e}(\vec{\omega})=\frac{H(\vec{\omega})+H(-\vec{\omega})}{2} \\
& H_{o}(\vec{\omega})=\frac{H(\vec{\omega})-H(-\vec{\omega})}{2 j} .
\end{aligned}
$$


To find the response of the EVEN and ODD neurons, we first note that

$$
X(\vec{\omega})=X_{e}(\vec{\omega})+j X_{o}(\vec{\omega}) .
$$

Since $x_{e}$ and $x_{o}$ are real, $X_{e}^{*}(-\vec{\omega})=X_{e}(\vec{\omega})$ and $X_{0}^{*}(-\vec{\omega})=X_{o}(\vec{\omega})$, where the * superscript denotes complex conjugation. This implies that

$$
X^{*}(-\vec{\omega})=X_{e}^{*} \vec{\omega}-j X_{o}^{*}(\vec{\omega})=X_{e}(\vec{\omega})-j X_{o}(\vec{\omega}) .
$$

Combining (6) and (7), we obtain

$$
\begin{aligned}
& X_{e}(\vec{\omega})=\frac{X(\vec{\omega})+X^{*}(-\vec{\omega})}{2} \\
& X_{o}(\vec{\omega})=\frac{X(\vec{\omega})+X^{*}(-\vec{\omega})}{2 j}
\end{aligned}
$$

Expanding (4) to $X(\vec{\omega})=\left(H_{e}(\vec{\omega})+j H(\vec{\omega})\right)\left(U_{e}(\vec{\omega})+j U_{o}(\vec{\omega})\right)$, and substituting into the equation above we obtain

$$
\begin{aligned}
& X_{e}(\vec{\omega})=H_{e}(\vec{\omega}) U_{e}(\vec{\omega})-H_{o}(\vec{\omega}) U_{o}(\vec{\omega}) \\
& X_{o}(\vec{\omega})=H_{o}(\vec{\omega}) U_{e}(\vec{\omega})+H_{e}(\vec{\omega}) U_{o}(\vec{\omega})
\end{aligned}
$$

In the feedforward system, the input to each chip is supplied by the silicon retina alone. Thus, $U_{e}(\vec{\omega})=R(\vec{\omega})$ and $U_{o}(\vec{\omega})=$ 0 , where $R(\vec{\omega})$ is the discrete spatial Fourier transform of the SUSTAINED silicon retina output $r(m, n)$, which is differentially encoded on ON and OFF channels. Thus, the responses of the EVEN and ODD neurons are $X_{e}(\vec{\omega})=H_{e}(\vec{\omega}) R(\vec{\omega})$ and $X_{o}(\vec{\omega}$ )$=H_{o}(\vec{\omega}) R(\vec{\omega})$. If the retinal input is a sine-wave grating with frequency $\vec{\omega}$, then the responses of the neurons will be proportional to $\left|H_{e}(\vec{\omega})\right|$ and $\left|H_{o}(\vec{\omega})\right|$.

For the feedback system, the input to each Gabor chip is the residual signal

$$
U(\vec{\omega})=R(\vec{\omega})-\beta \Sigma_{\phi} X_{\phi}(\vec{\omega})
$$

where $X_{\phi}$ denotes the output of the Gabor chip tuned to orientation $\phi$ and $\beta$ is a feedback factor which determines how much of the summed output is subtracted from the input in computing the residual. From (4), the output of the Gabor chip tuned to $\theta$ is

$$
X_{\theta}(\vec{\omega})=H_{\theta}(\vec{\omega})\left(R(\vec{\omega})-\beta \Sigma_{\phi} X_{\phi}(\vec{\omega})\right) .
$$

Summing over $\theta$, we find that

$$
\Sigma X_{\phi}(\vec{\omega})=\frac{\Sigma_{\phi} H_{\phi}(\vec{\omega})}{1+\beta \Sigma_{\phi} H_{\phi}(\vec{\omega})} R(\vec{\omega})
$$

Combining (8) and (9), we obtain $X_{\theta}(\vec{\omega}) H_{\theta}^{c}(\vec{\omega}) R(\vec{\omega})$ where the "c" superscript denotes the transfer function from the retinal input to the chip under coupling and

$$
H_{\theta}^{c}(\vec{\omega})=\frac{H_{\theta}(\vec{\omega})}{1+\beta \Sigma_{\phi} H_{\phi}(\vec{\omega})}
$$

We predict the responses of EVEN and ODD neurons using equations similar to those used for the feedforward system, except $H_{\theta}$ is replaced by $H_{\theta}^{c}$.

\section{REFERENCES}

[1] C. A. Mead, "Neuromorphic electronic systems," Proc. IEEE, vol. 78, pp. 1629-1636, 1990

[2] Seeing, Handbook of Perception and Cognition, 2nd ed., K. K. De Valois, Ed., Academic Press, San Diego, CA, 2000, pp. 79-128.

[3] J. M. Hupe, A. C. James, B. R. Payne, S. G. Lomber, P. Girard, and J. Bullier, "Cortical feedback improves discrimination between figure and background by V1, V2, and V3 neurons," Nature, vol. 394, pp. 784-787, 1998.

[4] D. H. Hubel and T. N. Wiesel, "Receptive fields, binocular interaction, and functional architecture in the cat's visual cortex," J. Physiol., vol. 160, pp. 106-154, 1962.

[5] A. B. Watson and J. A. J. Ahumada, "Model of human visual-motion sensing," J. Opt. Soc. Amer. A, vol. 2, pp. 322-342, Feb. 1985.

[6] E. H. Adelson and J. R. Bergen, "Spatiotemporal energy models for the perception of motion," J. Opt. Soc. Amer. A, vol. 2, pp. 284-299, Feb. 1985.

[7] I. Ohzawa, G. C. DeAngelis, and R. D. Freeman, "Stereoscopic depth discrimination in the visual cortex: Neurons ideally suited as disparity detectors," Science, vol. 249, pp. 1037-1041, Aug. 31, 1990.

[8] K. A. Boahen, "A burst-mode word-serial address-event link-I: Transmitter design," IEEE Trans. Circuits Syst. I, Fundam. Theory Appl., vol. 51, pp. 1269-1280, 2004.

[9] _ , "A burst-mode word-serial address-event link-II: Receiver design," IEEE Trans. Circuits Syst. I, Fundam. Theory Appl., vol. 51, pp. 1281-1291, 2004

[10] — "A burst-mode word-serial address-event link-III: Analysis and test results," IEEE Trans. Circuits Syst. I, Fundam. Theory Appl., vol. 51, pp. 1292-1300, 2004.

[11] T. Y. W. Choi, B. E. Shi, and K. Boahen, "An on-off orientation selective address-event representation image transceiver chip," IEEE Trans. Circuits Syst. I, Fundam. Theory Appl., vol. 51, no. 2, pp. 342-353, 2004.

[12] G. Cauwenberghs and J. Waskiewicz, "Focal-plane analog VLSI cellular implementation of the boundary contour system," IEEE Trans. Circuits Syst. I, Fundam. Theory Appl., vol. 46, no. 2, pp. 327-334, Feb. 1999.

[13] S. C. Liu, J. Kramer, G. Indiveri, T. Delbruck, T. Burg, and R. Douglas, "Orientation selective aVLSI spiking neurons," Neural Networks, vol. 14, pp. 629-643, 2001.

[14] B. E. Shi, E. K. C. Tsang, and P. S. P. Au, "An ON/OFF temporal filter circuit for visual motion analysis," in Proc. IEEE Int. Symp. Circuits and Systems, vol. 3, Vancouver, BC, Canada, May 2004, pp. 85-88.

[15] E. K. C. Tsang and B. E. Shi, "A preference for phase-based disparity in a neuromorphic implementation of the binocular energy model," Neural Computation, vol. 16, no. 8, pp. 1579-1600, Aug. 2004.

[16] T. Roska and L. O. Chua, "The CNN universal machine: An analogic array computer," IEEE Trans. Circuits Syst. II, Analog Digit. Signal Process., vol. 40, no. 2, pp. 163-173, Feb. 1993.

[17] V. Gruev and R. Etienne-Cummings, "Implementation of steerable spatiotemporal image filters on the focal plane," IEEE Trans. Circuits Syst. II, Analog Digit. Signal Process., vol. 49, no. 3, pp. 233-244, Mar. 2002.

[18] T. Serrano-Gotarredona, A. G. Andreou, and B. Linares-Barranco, "AER image filtering architecture for vision-processing systems," IEEE Trans. Circuits Syst. I, Fundam. Theory Appl., vol. 46, no. 9, pp. 1064-1071, Sep. 1999.

[19] P. Venier, A. Mortara, X. Arreguit, and E. A. Vittoz, "An integrated cortical layer for orientation enhancement," IEEE J. Solid-State Circuits, vol. 32, no. 2, pp. 177-186, Feb. 1997.

[20] D. L. Standley, "An object position and orientation IC with embedded imager," IEEE J. Solid-State Circuits, vol. 26, no. 6, pp. 1853-1859, Dec. 1991.

[21] P. F. Ruedi, P. Heim, F. Kaess, E. Grenet, F. Heitger, P. Y. Burgi, S. Gyger, and P. Nussbaum, "A $128 * 128$ pixel $120 \mathrm{~dB}$ dynamic range vision sensor chip for image contrast and orientation extraction," IEEE J. Solid-State Circuits, vol. 38, no. 12, pp. 2325-2333, Dec. 2003. 
[22] K. A. Zaghloul and K. Boahen, "Optic nerve signals in a neuromorphic chip I: Outer and inner retina models," IEEE Trans. Biomed. Eng., vol. 51, no. 4, pp. 657-666, Apr. 2004.

[23] - "Optic nerve signals in a neuromorphic chip II: Testing and results," IEEE Trans. Biomed. Eng., vol. 51, no. 4, pp. 667-675, Apr. 2004

[24] B. E. Shi, "Focal plane implementation of 2-D steerable and scalable cortical filters," J. VLSI Signal Process., vol. 23, pp. 319-334, 1999.

[25] D. G. Albrecht and W. S. Geisler, "Motion selectivity and the contrast response function of simple cells in the visual cortex," Visual Neurosci., vol. 7, pp. 531-546, 1991.

[26] D. J. Heeger, "Normalization of cell responses in cat striate cortex," $\mathrm{Vi}$ sual Neurosci., vol. 9, pp. 181-197, 1992.

[27] - "Half-squaring in responses of cat striate cells," Visual Neurosci., vol. 9, pp. 427-443, 1992.

[28] J. G. Daugman, "Two-dimensional spectral analysis of cortical receptive field profiles," Vision Res., vol. 20, pp. 847-856, 1980.

[29] J. P. Jones and L. A. Palmer, "An evaluation of the two-dimensional Gabor filter model of simple receptive fields in cat striate cortex," $J$. Neurosci., vol. 58, no. 6, pp. 1233-1258, Dec. 1987.

[30] D. A. Pollen and S. F. Ronner, "Phase relationships between adjacent simple cells in the visual cortex," Science, vol. 212, pp. 1409-1411, 1981.

[31] K. A. Boahen, "Point-to-point connectivity between neuromorphic chips using address events," IEEE Trans. Circuits Syst. II, Analog Digit. Signal Process., vol. 47, no. 5, pp. 416-434, May 2000.

[32] D. Ferster and K. D. Miller, "Neural mechanisms of orientation selectivity in the visual cortex," Ann. Rev. Neurosci., vol. 23, pp. 441-471, 2000.

[33] B. E. Shi and K. Boahen, "Competitively coupled orientation selective cellular neural networks," IEEE Trans. Circuits Syst. I, Fundam. Theory Appl., vol. 49, no. 3, pp. 388-394, Mar. 2002.

[34] K. V. Mardia, Statistics of Directional Data. London, U.K.: Academic, 1972.

[35] R. J. Douglas, C. Koch, M. Mahowald, K. A. Martin, and H. H. Suarez, "Recurrent excitation in neocortical circuits," Science, vol. 269, pp. 981-985, 1995.

[36] V. Dragoi, J. Sharma, and M. Sur, "Adaptation-induced plasticity of orientation tuning in adult visual cortex," Neuron, vol. 28, pp. 287-298, 2000.

[37] A. Schoups, R. Vogels, N. Qian, and G. Orban, "Practising orientation identification improves orientation coding in V1 neurons," Nature, vol. 412, pp. 549-553, 2001.

[38] A. F. Teich and N. Qian, "Learning and adaptation in a recurrent model of V1 orientation selectivity," J. Neurophysiol., vol. 89, pp. 2086-2100, 2003.

[39] T. W. Troyer, A. E. Krukowski, N. J. Priebe, and K. D. Miller, "Contrast-invariant orientation tuning in cat visual cortex: Thalamocortical input tuning and correlation-based intracortical connectivity," J. Neurosci., vol. 18, pp. 5908-5927, 1998.

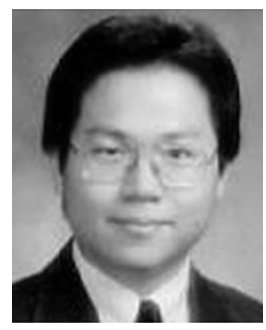

Thomas Yu Wing Choi (S'03) was born in Hong Kong in 1975. He received the B.S., M.S., and Ph.D. degrees in electrical and electronic engineering from the Hong Kong University of Science and Technology, Hong Kong, in 1997, 1999, and 2003, respectively.

He is currently employed by Hong Kong Science and Technology Research Institute Limited as an Analog Integrated Circuit Design Engineer. His research interests include analog very large-scale integration design and neural networks.

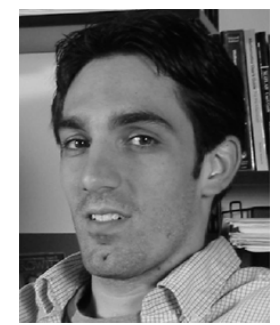

Paul A. Merolla received the B.S. degree with high distinction in electrical engineering from the University of Virginia, Charlottesville, in 2000. He is currently working toward the Ph.D. degree in bioengineering at the University of Pennsylvania, Philadelphia.

His research interests include very large-scale integration models of cortical networks, vision systems, and asynchronous digital interfaces for routing, and interchip connectivity.

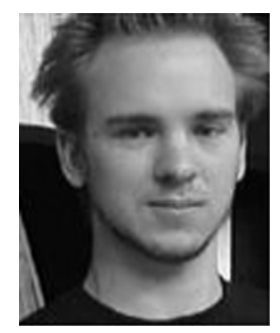

John V. Arthur received the B.S.E. degree (summa cum laude) in electrical engineering from Arizona State University, Phoenix, in 2000. He is currently working toward the Ph.D. degree in bioengineering at the University of Pennsylvania.

His research interests include mixed-mode very large-scale integration, neuromorphic learning systems, and asynchronous interchip communication.

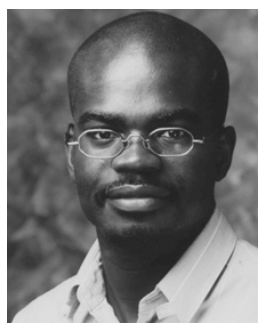

Kwabena A. Boahen B.S. and M.S.E. degrees in electrical and computer engineering from the Johns Hopkins University, Baltimore MD, in the concurrent masters-bachelors program, both in 1989, and the Ph.D. degree in computation and neural systems from the California Institute of Technology (Caltech), Pasadena, CA in 1997. He received the

$\mathrm{He}$ is an Associate Professor in the Bioengineering Department, University of Pennsylvania, Philadelphia, where he holds a secondary appointment in Electrical Engineering. His current research interests include mixed-mode multichip very large-scale integration models of biological sensory and perceptual systems, and their epigenetic development, and asynchronous digital interfaces for interchip connectivity.

Dr. Boahen held a Sloan Fellowship for Theoretical Neurobiology while at Caltech. He was awarded a Packard Fellowship in 1999, a National Science Foundation CAREER Award in 2001, and an Office of Naval Research YIP in 2002. He is a Member of Tau Beta Kappa.

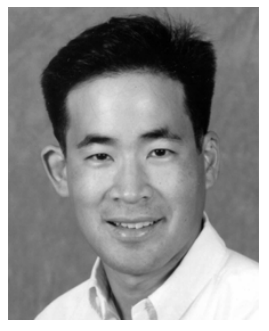

Bertram E. Shi received the B.S. and M.S. degrees in electrical engineering from Stanford University, Stanford, CA, and the Ph.D. degree in electrical engineering from the University of California at Berkeley, in 1987, 1988, and 1994, respectively.

He then joined the faculty of the Department of Electrical and Electronic Engineering at the Hong Kong University of Science and Technology, where he is currently an Associate Professor. His research interests are in analog very large-scale integration and cellular neural networks, bio-inspired and neuromorphic engineering, machine vision, and image processing.

Dr. Shi's IEEE activities have included Student Activities Chair of the IEEE Hong Kong Section, Associate Editor for the IEEE TRANSACTIONS ON CIRCUITS AND SySTEMS-I: FundAMENTAL THEORY AND APPLICATIONS, Secretary and Chair-elect for the IEEE Circuits and Systems Society Technical Committee on Cellular Neural Networks, and Array Computing and Distinguished Lecturer for the IEEE Circuits and Systems Society. 